\title{
Tanshinone IIA potentiates the efficacy of 5-FU in Colo205 colon cancer cells in vivo through downregulation of P-gp and LC3-II
}

\author{
CHIN-CHENG SU \\ Department of Surgery, Changhua Christian Hospital, Changhua, Changhua 500-06; \\ Mingdao University, Pee-Tow, Changhua 52345; School of Chinese Medicine, \\ College of Chinese Medicine, China Medical University, Taichung 40402, Taiwan, R.O.C.
}

Received September 18, 2011; Accepted December 19, 2011

DOI: $10.3892 /$ etm.2011.441

\begin{abstract}
Traditional Chinese herbal medicines are widely accepted as an option for the treatment of colorectal cancers. Danshen (Salviae miltiorrhizae Radix) is widely prescribed in traditional Chinese medicine for cardiovascular diseases. Tanshinone IIA (Tan-IIA) is extracted from Danshen. Our previous studies have shown that Tan-IIA induces apoptosis in Colo205 human colon cancer cells in vitro and in vivo. In the present study, we investigated the efficacy of Tan-IIA and 5-fluorouracil (5-FU) in a Colo205 cell xenograft model. For in vivo studies, SCID mice were engrafted with Colo205 cells and from day 10 onwards were randomly divided into 3 groups and treated with 5-FU plus Tan-IIA, 5-FU plus corn oil, and the vehicle alone. At the end of a 4-week dosing schedule, the SCID mice were sacrificed and xenograft tumors were dissected for protein western blot analysis. Our results showed that the Colo205 xenograft model co-treated with Tan-IIA plus 5-FU caused a reduction in the xenograft tumor volumes and decreased P-glycoprotein (P-gp) and microtubule-associated protein light chain 3 (LC3)-II expression compared to 5-FU alone. Based on these observations, it may be possible to develop Tan-IIA plus 5-FU as therapeutic agents for human colon cancer.
\end{abstract}

\section{Introduction}

Colorectal cancer is the third leading cause of cancer-related mortality in Taiwan (1) and the second leading cause of cancer-related mortality in Western countries $(2,3)$. Traditional Chinese herbal medicines are widely accepted as an option for the treatment of colorectal cancers and there are intense

Correspondence to: Dr Chin-Cheng Su, Changhua Christian Hospital, 135 Nan-Hsiao Street, Changhua, Changhua 500-06, Taiwan, R.O.C.

E-mail:succ.maeva@msa.hinet.net

Key words: tanshinone IIA, 5-fluorouracil, Colo205, P-glycoprotein, microtubule-associated protein light chain $3 \mathrm{II}$, in vivo efforts under way to identify new herbs and bioactive pure compounds (4,5). Danshen (Salviae miltiorrhizae Radix) is widely prescribed in traditional Chinese medicine for cardiovascular diseases $(6,7)$. Tanshinone IIA (Tan-IIA; $\mathrm{C}_{19} \mathrm{H}_{18} \mathrm{O}_{3}$ ) is extracted from Danshen $(8,9)$, and possesses anti-inflammatory $(10,11)$ and antioxidant properties $(12,13)$. Our previous studies have shown that Tan-IIA inhibits growth and induces apoptosis in Colo205 human colon cancer cells (14) and MDA-MB-231 breast cancer cells in vitro (15). 5-Fluorouracil (5-FU) is one of the chemotherapeutic medicines for colon cancer, but has low efficacy (16). To increase its therapeutic potential, there is interest in combining it with another medicine. The efficacy of Tan-IIA plus 5-FU in human colon cancer cells has not been established. Accordingly, we investigated the effects of Tan-IIA and 5-FU in a Colo205 human colon cancer cell xenograft model.

\section{Materials and methods}

Materials. Tan-IIA (purity $>96 \%$; HPLC) was purchased from Herbasin Co. (China). Aprotinin, antipain, sodiumdeoxycholate, leupeptin, sodium orthovanadate, Triton X-100 and Tris- $\mathrm{HCl}$ were obtained from Sigma-Aldrich (St. Louis, MO, USA). Dimethyl sulfoxide (DMSO), potassium phosphate and TE buffer were purchased from Merck KGaA (Darmstadt, Germany). RPMI-1640 medium and fetal bovine serum (FBS) were obtained from Gibco BRL (Grand Island, NY, USA). Antibodies to P53, P21, VEGF, MMP-2 and -7, Topoisomerase I, Erb-B2, P-glycoprotein (P-gp), LC-3II, Bcl-2 and $\beta$-actin were obtained from Sigma-Aldrich. Penicillinstreptomycin, Trypsin-EDTA and glutamine were obtained from Gibco BRL. Sodium dodecylsulfate polyacrylamide gel and electrophoresis (SDS-PAGE) running buffer (10X), Tris, Tween-20, SDS and 5X TBE buffer were obtained from Amresco (St. Louis, MO, USA). BioMax film was obtained from Kodak.

Cell cultures. The Colo205 human colon cancer cell line was obtained from the Food Industry Research and Development Institute (Hsin-chu, Taiwan). The cells were placed into tissue culture flasks $\left(75 \mathrm{~cm}^{2}, 250 \mathrm{ml}\right)$ and grown at $37^{\circ} \mathrm{C}$ in humidified $5 \% \mathrm{CO}_{2}$ and $95 \%$ air atmosphere in RPMI-1640 medium containing $10 \%$ heat-inactivated FBS, $1 \%$ HEPES, $1 \%$ sodium 
pyruvate, $1 \%$ glutamine and $2 \%$ penicillin-streptomycin (10,000 U/ml penicillin; $10 \mathrm{mg} / \mathrm{ml}$ streptomycin).

In vivo tumor xenograft study. In the present study, 5-weekold male nude SCID mice (24 in total) were xenografted with Colo205 colon cancer cells $\left(3 \times 10^{6} / 0.2 \mathrm{ml}\right)$ and maintained in a pathogen-free environment (Laboratory Animal Center of Tzu Chi University, Hualien, Taiwan). From day 10, SCID mice bearing Colo205 human colon cancer cell engrafts were divided randomly into 3 groups and then treated with 5 -FU $(20 \mathrm{mg} / \mathrm{kg}$, every week on day 1) plus Tan-IIA $(20 \mathrm{mg} / \mathrm{kg}$, every week on days 3 and 5), 5-FU (20 mg/kg, every week on day 1) plus corn oil (every week on days 3 and 5) or the vehicle alone (normal saline, every week on day 1 and corn oil every week on days 3 and 5) (Fig. 1). Following xenograft transplantation, mice exhibiting tumors were monitored and tumor size was measured once every 3 days using calipers. The tumor volume in each animal was estimated according to the formula: tumor volume $\left(\mathrm{mm}^{3}\right)=\mathrm{L} \times \mathrm{W} 2 / 2$ (where $\mathrm{L}$ is the length and $\mathrm{W}$ is the width) with the final measurement taken 4 weeks after tumor cell inoculation. At the end of the 4-week dosing schedule, the SCID mice were sacrificed with $\mathrm{CO}_{2}$ inhalation, xenograft tumors were dissected and total protein extracted for western blot analysis. The animal use protocol has been reviewed and approved by the Institutional Animal Care and Use Comittee (IACUC) Board, TZU-CHI Hospital (IACUC Approval No: 97-21).

Synergistic effects of Tan-IIA and 5-FU on the protein expression of $p 53, p 21, V E G F, M M P-2$ and -7 , topoisomerase I, Erb-B2, P-gp, LC-3II, Bcl-2 and $\beta$-actin in Colo205 cell xenograft tumors

Protein preparation. Total proteins were extracted as previously described (17). Briefly, the xenograft tumors were broken into pieces and the resulting thick liquid was then suspended in modified PRO-PREP ${ }^{\mathrm{TM}}$ buffer (iNtRON Biotechnology Inc., Korea) for $40 \mathrm{~min}$ at $4^{\circ} \mathrm{C}$. Lysates were immediately centrifuged at $13,000 \mathrm{x}$ g for $20 \mathrm{~min}$ at $4^{\circ} \mathrm{C}$, and the supernatant was collected, aliquated $(20 \mu 1 /$ tube $)$ and

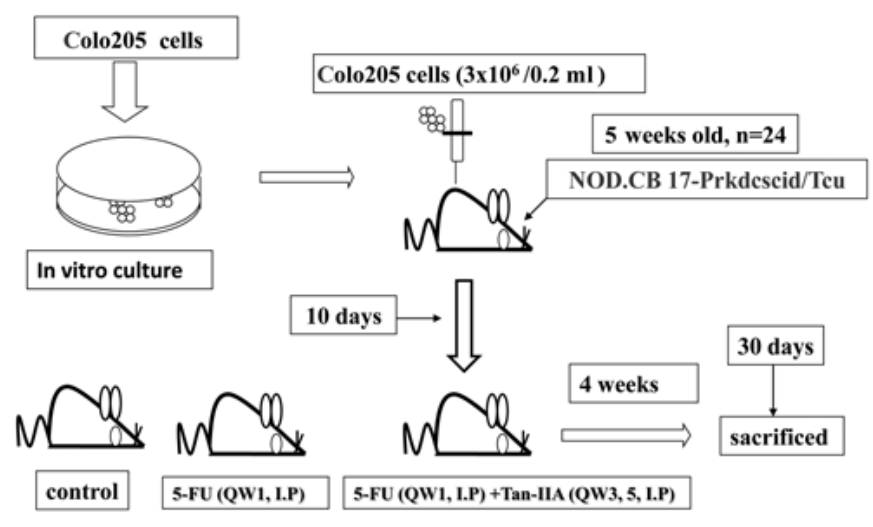

Figure 1. Colo205 human colon cancer cell xenograft tumors were treated with 5-FU or 5-FU plus Tan-IIA for 4 weeks. Controls were separately treated with normal saline and corn oil. 5-FU, 5-fluorouracil; Tan-IIA, tanshinone IIA; QW1, every week on day 1; QW3,5, every week on days 3 and 5; I.P., intraperitoneally.

stored at $-80^{\circ} \mathrm{C}$ until assay. The extracted protein concentrations were measured using the Bradford method (18).

Western blot analysis. All protein samples were separated by $8-15 \%$ SDS-PAGE as previously described (17). The SDS-separated proteins were equilibrated in transfer buffer (25 mM Tris, pH 8.5, 0.2 M glycine and 20\% methanol) and transferred onto a PVDF membrane (Millipore, Bedford, MA, USA). The membranes were incubated with 5\% non-fat dry milk in Tris-buffered saline containing $0.1 \%$ Tween- 20 for $1 \mathrm{~h}$. These membranes were then washed and incubated with appropriate dilutions of specific antibodies at $4^{\circ} \mathrm{C}$ overnight [including p53 (1:2000, MAB 1355; R\&D Systems), p21 (1:2000, MAB 1047; R\&D Systems), VEGF (1:500, V4758; Sigma), MMP-2 (1:200, MAB 902; R\&D Systems) and MMP-7 (1:500, MAB 9071; R\&D Systems), topoisomerase I (1:1000, T8573; Sigma), Erb-B2 (1:500, MAB 1129; R\&D Systems), P-gp (1:500, P7965; Sigma), microtubule-associated
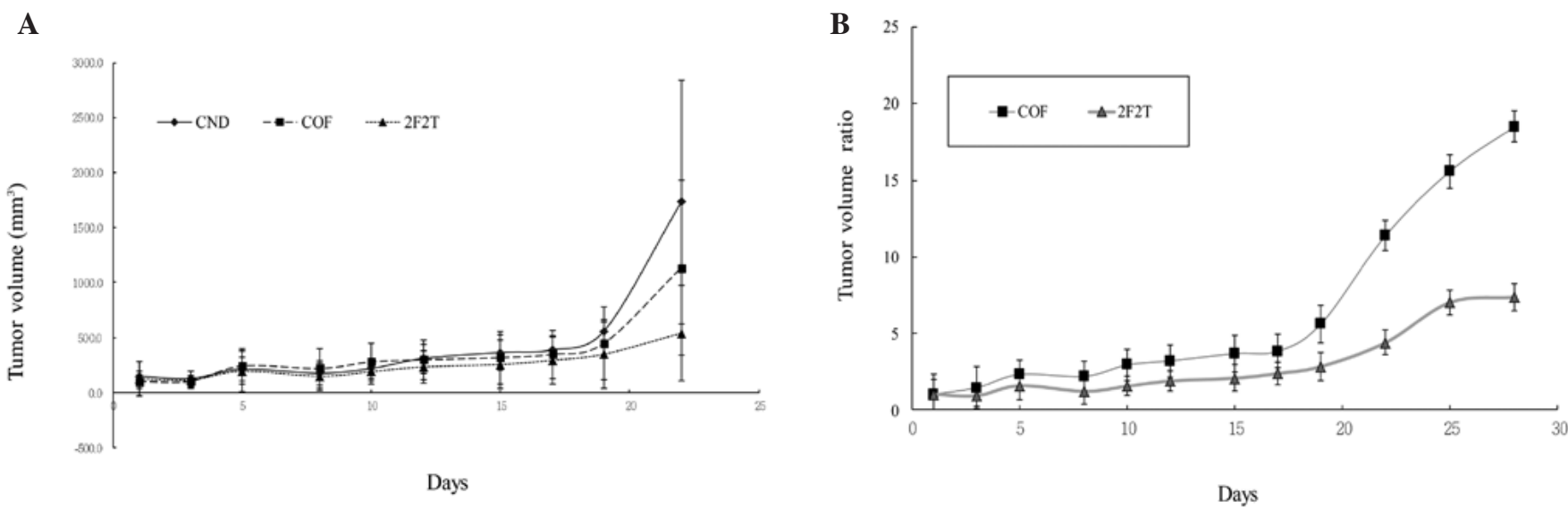

Figure 2. SCID mice bearing Colo205 human colon cancer cell xenografts were divided randomly into 3 groups and then treated with 5-FU plus Tan-IIA (2F2T group); 5-FU only (COF group) or vehicle alone (CND group). The tumor volumes were measured every 3 days. The tumor volume in each animal was estimated according to the formula: tumor volume $\left(\mathrm{mm}^{3}\right)=\mathrm{L}$ x W2/2 (where $\mathrm{L}$ is the length and W is the width) with the final measurement taken 4 weeks after tumor cell inoculation. (A) The results showed that Tan-IIA could potentiate the growth inhibitory effect of 5-FU in a colon cancer nude mouse model. (B) Our results showed that co-treatment with Tan-IIA plus 5-FU in the Colo205 xenograft model caused a reduction in the xenograft tumor volumes when compared to treatment with 5-FU only. 
A
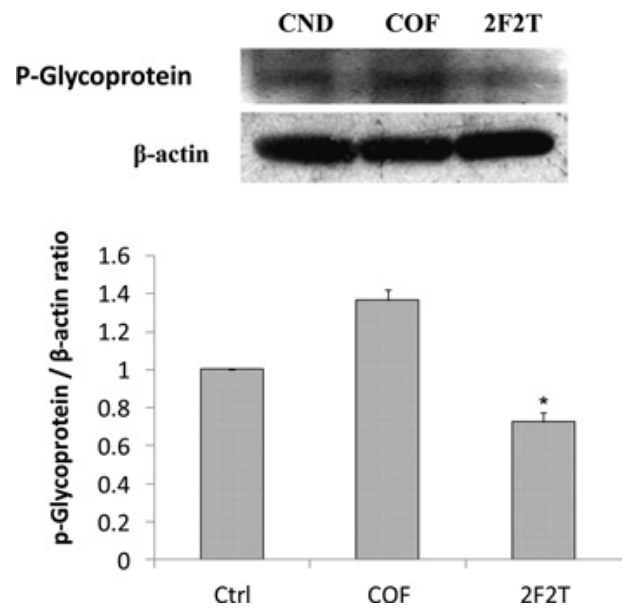

C
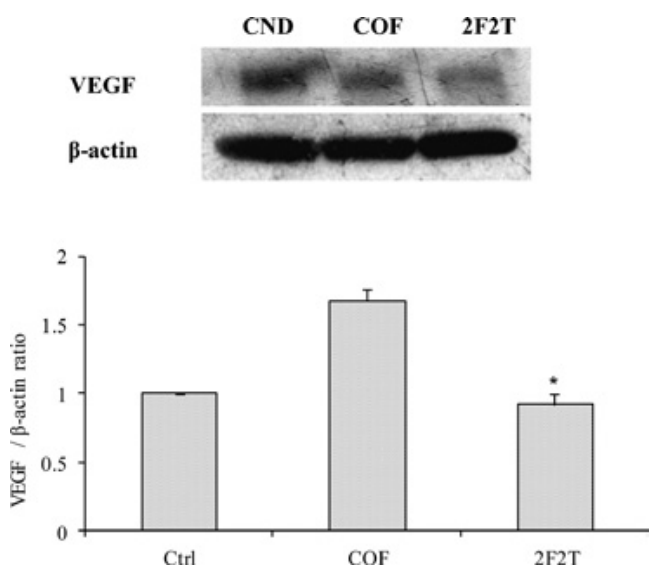

$\mathbf{E}$
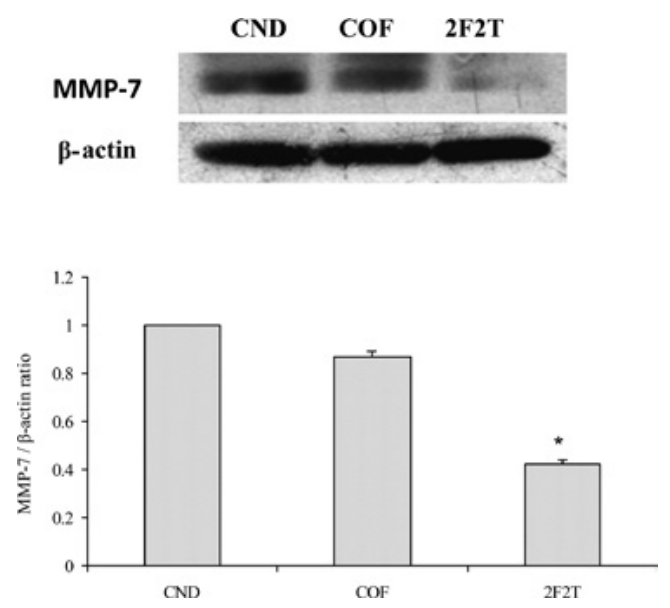

protein light chain 3 (LC3)-II (1:1500, L7543; Sigma), Bcl-2 (1:500; R\&D Systems) and $\beta$-actin (1:15000, A5441, Sigma)]. Following incubation with anti-mouse peroxidase-conjugated antibody (1:15000) (Sigma-Aldrich) the immunoreactive bands were visualized with an enhanced chemiluminescence (ECL, Millipore) detection kit. The detection of $\beta$-actin was used as an internal control in all of the data for western blot analysis. Immunoreactive bands were scanned (GS-800; Bio-Rad Life Science Products, Hercules, CA, USA) and analyzed using a digital scanning densitometer (Quantity One, v4.4.0; Bio-Rad Life Science Products).

Statistical analysis. Values were presented as the means \pm SD. The Student's t-test was used to analyze statistical significance.
B
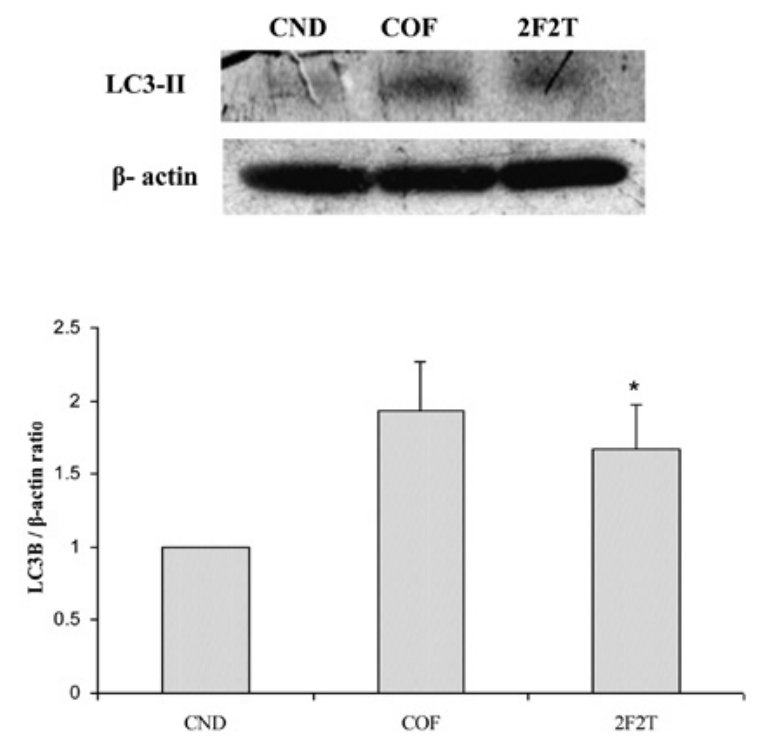

D
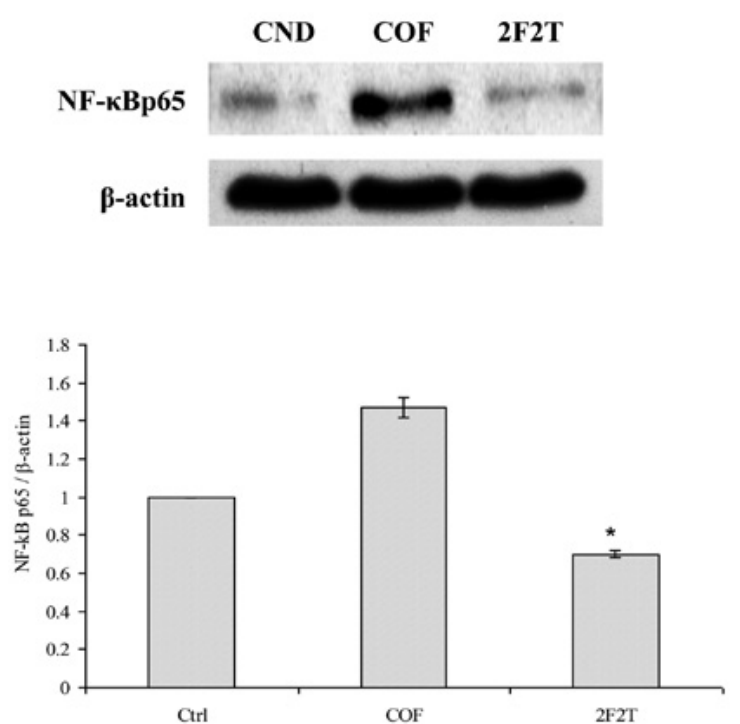

Figure 3. Our results showed that co-treatment with Tan-IIA plus 5-FU in the Colo205 xenograft model decreased the protein expressions of (A) P-gp,

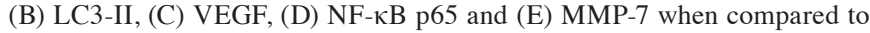
5 -FU only. Values are expressed as the means \pm SD. *Significant difference at $\mathrm{p}<0.05,5$-FU plus Tan-IIA vs. the respective group without Tan-IIA treatment.

$\mathrm{P}<0.05$ was considered to indicate statistically significant differences for all tests.

\section{Results}

Synergistic effects of Tan-IIA and 5-FU in the Colo205 cell xenograft tumor model. SCID mice bearing Colo205 human colon cancer cell xenografts were divided randomly into 3 groups and then treated with 5-FU plus Tan-IIA, 5-FU only, or the vehicle alone. The tumor volumes and SCID mouse body weights were measured every 3 days. The xenograft tumor volumes were measured. The tumor volumes were $1,733.20 \pm 1,113.68$, $1,134 \pm 795.95$ and $537.42 \pm 437.36 \mathrm{~mm}^{3}$ for the control, 5-FU, and 5-FU plus Tan-IIA groups, respectively. The tumor volume 
enlarged ratios were $11.82 \pm 0.64,11.5 \pm 0.7$ and $4.38 \pm 0.31$ for the control, 5-FU, and 5-FU plus Tan-IIA groups, respectively. The results showed that Tan-IIA could potentiate the effect of 5-FU in a colon cancer nude mouse model (Fig. 2A and B).

Effects of Tan-IIA and 5-FU on the protein expressions of p53, p21, VEGF, MMP-2 and -7, topoisomerase I, Erb-B2, $P$-gp, LC-3II, Bcl-2 and $\beta$-actin in Colo205 cell xenograft tumors. SCID mice bearing Colo205 human colon cancer cell xenograft tumors were divided randomly into 3 groups and then treated with 5-FU plus Tan-IIA, 5-FU only, or the vehicle alone. At the end of the 4 -week dosing schedule, the SCID mice were sacrificed by $\mathrm{CO}_{2}$ inhalation and xenograft tumors dissected with total protein extracted for western blot analysis. The results showed that SCID mice with Colo205 cell xenograft tumors treated with 5-FU [20 mg/kg intraperitoneally (i.p.), every week on day 1] plus Tan-IIA ( $20 \mathrm{mg} / \mathrm{kg}$ i.p., every week on days 3 and day 5$)$ had downregulated P-gp (Fig. 3A), LC-3II (Fig. 3B), VEGF (Fig. 3C), NF-kB p65 (Fig. 3D) and MMP-7 (Fig. 3E) expression when compared to the group treated only with 5-FU.

\section{Discussion}

$\mathrm{P}-\mathrm{gp}$ is a cell membrane-associated protein, with high expression in cancer tissue, which functions as a drug export pump that decreases intracellular concentrations of chemotherapeutic agent $(19,20)$. Our results showed that 5-FU plus Tan-IIA decreased the protein expression of P-gp when compared to the 5-FU group. It was documented that simultaneous and continuous exposure of colon cancer cells to low concentrations of 5-FU for $144 \mathrm{~h}$ showed a strong antagonism in vitro and that 14-days of continuous infusion of 5-FU significantly stimulated angiogenesis in vivo $(21,22)$ Our results also showed that 5-FU increased the protein expression of VEGF when compared to the control group. The 5-FU plus Tan-IIA group decreased the protein expression of VEGF when compared to the 5-FU group. It has been well documented that LC3-II can be used as a marker of autophagy and can be detected using western blot analysis $(23,24)$. Our results showed that the combination treatment of Tan-IIA plus 5-FU decreased the protein expression of LC3-II when compared to the 5-FU group. The combination treatment of autophagy inhibitor plus 5-FU significantly increased apoptotic cell death. 5-FU-induced apoptosis in colon cancer cells can be enhanced by the inhibition of autophagy (21). Elevated MMP-7 protein expression was a predictor of colon cancer recurrence and liver metastasis. High expression of MMP-7 was related to decreased survival (25). It has been well documented that the combination of gambogic acid and celastrol has a synergistic antitumor effect. The effect can be attributed to apoptosis induced by a decrease in NF- $\mathrm{BB}$ pathway activation, therefore, NF- $\mathrm{KB}$ p65 protein may be involved $(26,27)$. The inhibition of NF- $\mathrm{kB}$ augments sensitivity to 5-FU/folinic acid in colon cancer (28). Our results showed that Tan-IIA plus 5-FU decreased the protein expression of MMP-7 and NF- $\mathrm{KB}$ p65 when compared to the 5-FU group. Tan-IIA plus 5-FU has a synergistic effect on Colo205 cell xenograft tumors. The effect can be primarily attributed to a decrease in MMP-7 and NF- $\mathrm{\kappa B}$ p65 expression. These observations suggest that
Tan-IIA potentiates the efficacy of 5-FU in a colon cancer nude SCID mouse model through downregulating P-gp, LC3-II, VEGF, MMP-7 and NF- $\mathrm{BB}$ p65 protein expression. This is the first report of 5-FU plus Tan-IIA downregulating P-gp, LC3-II, VEGF, MMP-7 and NF-אB p65 protein expression in vivo. The use of Tan-IIA may be a promising strategy for the adjuvant chemotherapy of colon cancer.

\section{Acknowledgements}

This study was supported by a grant (CCMP97-RD-041) from the Committee on Chinese Medicine and Pharmacy, Department of Health, Executive Yuan, Taiwan, R.O.C.

\section{References}

1. Department of Health, Executive Yuan, Taipei, Taiwan R.O.C: Statistics of Causes of Death, 2007. p33, 2008.

2. Jemal A, Tiwari RC, Murray T, et al: Cancer statistics, 2004. CA Cancer J Clin 54: 8-29, 2004.

3. Wei SC, Su YN, Tsai-Wu JJ, et al: Genetic analysis of the APC gene in Taiwanese familial adenomatous polyposis. J Biomed Sci 11: 260-265, 2004.

4. Verhoef MJ, Balneaves LG, Boon HS and Vroegindewey A: Reasons for and characteristics associated with complementary and alternative medicine use among adult cancer patients: a systematic review. Integr Cancer Ther 4: 274-286, 2005.

5. Boon $\mathrm{H}$ and Wong J: Botanical medicine and cancer: a review of the safety and efficacy. Expert Opin Pharmacother 5: 2485-2501, 2004.

6. Fish JM, Welchons DR, Kim YS, Lee SH, Ho WK and Antzelevitch C: Dimethyl lithospermate B, an extract of Danshen, suppresses arrhythmogenesis associated with the Brugada syndrome. Circulation 113: 1393-1400, 2006.

7. Chang PN, Mao JC, Huang SH, et al: Analysis of cardioprotective effects using purified Salvia miltiorrhiza extract on isolated rat hearts. J Pharmacol Sci 101: 245-249, 2006.

8. Che AJ, Zhang JY, Li CH, Chen XF, Hu ZD and Chen XG: Separation and determination of active components in Radix Salviae miltiorrhizae and its medicinal preparations by nonaqueous capillary electrophoresis. J Sep Sci 27: 569-575, 2004.

9. Zhou L, Zuo Z and Chow MS: Danshen: an overview of its chemistry, pharmacology, pharmacokinetics, and clinical use. J Clin Pharmacol 45: 1345-1359, 2005.

10. Jang SI, Kim HJ, Kim YJ, Jeong SI and You YO: Tanshinone IIA inhibits LPS induced NF-kappaB activation in RAW 264.7 cells: possible involvement of the NIK-IKK, ERK1/2, p38 and JNK pathways. Eur J Pharmacology 542: 1-7, 2006.

11. Li W, Li J, Ashok M, Wu R, et al: A cardiovascular drug rescues mice from lethal sepsis by selectively attenuating a lateacting pro-inflammatory mediator, high mobility group box 1 . J Immunology 78: 3856-3864, 2007.

12. Lin R, Wang WR, Liu JT, Yang GD and Han CJ: Protective effect of Tanshinone IIA on human umbilical vein endothelial cell injured by hydrogen peroxide and its mechanism. J Ethnopharmacol 108: 217-222, 2006.

13. Wang AM, Sha SH, Lesniak W and Schacht J: Tanshinone (Salviae miltiorrhizae extract) preparations attenuate aminoglycoside-induced free radical formation in vitro and ototoxicity in vivo. Antimicrob Agents Chemother 47: 1836-1841, 2003.

14. Su CC, Chen GW, Kang JC and Chan MH: Growth inhibition and apoptosis induction by tanshinone IIA in human colon adenocarcinoma cells. Planta Med 74: 1357-1362, 2008.

15. Su CC and Lin YH: Tanshinone IIA inhibits human breast cancer cells through increased Bax to Bcl-xL ratios. Int J Mol Med 22: 357-361, 2008.

16. Chau I, Norman AR, Cunningham D, et al: A randomised comparison between 6 months of bolus fluorouracil/leucovorin and 12 weeks of protracted venous infusion fluorouracil as adjuvant treatment in colorectal cancer. Ann Oncol 16: 549-557, 2005.

17. Chen HC, Hsieh WT, Chang WC and Chung JG: Aloe-emodin induced in vitro $\mathrm{G} 2 / \mathrm{M}$ arrest of cell cycle in human promyelocytic leukemia HL-60 cells. Food Chem Toxicol 42: 1251-1257, 2004. 
18. Bradford MM: A rapid and sensitive method for the quantitation of microgram quantities of protein utilizing the principle of protein-dye binding. Anal Biochem 72: 248-254, 1976.

19. Gottesman MM, Pastan I and Ambudkar SV: P-glycoprotein and multidrug resistance. Curr Opin Genet Dev 6: 610-617, 1996.

20. Lum BL, Gosland MP, Kaubisch S and Sikic BI: Molecular targets in oncology: implications of the multidrug resistance gene. Pharmacotherapy 13: 88-109, 1993.

21. Li J, Hou N, Faried A, Tsutsumi S, Takeuchi T and Kuwano H: Inhibition of autophagy by 3-MA enhances the effect of 5-FU-induced apoptosis in colon cancer cells. Ann Surg Oncol 16: 761-771, 2009.

22. Albertsson P, Lennernäs B and Norrby K: Low-dose continuous 5-fluorouracil infusion stimulates VEGF-A-mediated angiogenesis. Acta Oncol 48: 418-425, 2009.

23. Kirkegaard K, Taylor MP and Jackson WT: Cellular autophagy: Surrender, avoidance and subversion by microorganisms. Nat Rev Microbiol 2: 301-314, 2004
24. Eskelinen EL, Prescott AR, Cooper J, et al: Inhibition of autophagy in mitotic animal cells. Traffic 3: 878-893, 2002.

25. Fang YJ, Lu ZH, Wang GQ, Pan ZZ, Zhou ZW, Yun JP, Zhang MF and Wan DS: Elevated expressions of MMP7, TROP2, and survivin are associated with survival, disease recurrence, and liver metastasis of colon cancer. Int J Colorectal Dis 24: 875-884, 2009.

26. Xu WL, Liu JR, Liu HK, Qi GY, Sun XR, Sun WG and Chen BQ: Inhibition of proliferation and induction of apoptosis by gammatocotrienol in human colon carcinoma HT-29 cells. Nutrition 25: 555-566, 2009.

27. He D, Xu Q, Yan M, Zhang P, Zhou X, Zhang Z, Duan W, Zhong L, Ye D and Chen W: The NF-kappa B inhibitor, celastrol, could enhance the anti-cancer effect of gambogic acid on oral squamous cell carcinoma. BMC Cancer 9: 343, 2009.

28. Voboril R, Hochw SN, Li J, Brank A, Weberova J, Wessels F Moldawer LL, Camp ER and MacKay SL: Inhibition of NF-kappa B augments sensitivity to 5-fluorouracil/folinic acid in colon cancer. J Surg Res 120: 178-188, 2004. 